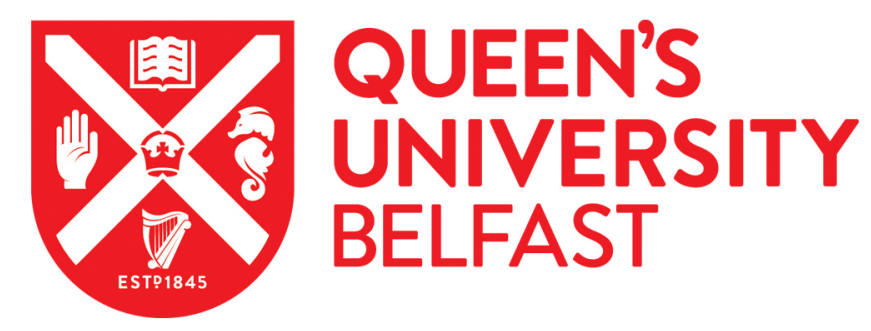

\title{
Untargeted metabolomic analysis of human serum samples associated with different levels of red meat consumption: A possible indicator of type 2 diabetes?
}

Carrizo, D., Chevallier, O. P., Woodside, J. V., Brennan, S. F., Cantwell, M. M., Cuskelly, G., \& Elliott, C. T. (2016). Untargeted metabolomic analysis of human serum samples associated with different levels of red meat consumption: A possible indicator of type 2 diabetes? Food Chemistry.

https://doi.org/10.1016/j.foodchem.2016.10.056

\section{Published in:}

Food Chemistry

\section{Document Version:}

Peer reviewed version

Queen's University Belfast - Research Portal:

Link to publication record in Queen's University Belfast Research Portal

\section{Publisher rights}

Copyright 2016 Elsevier.

This manuscript is distributed under a Creative Commons Attribution-NonCommercial-NoDerivs License

(https://creativecommons.org/licenses/by-nc-nd/4.0/), which permits distribution and reproduction for non-commercial purposes, provided the author and source are cited.

\section{General rights}

Copyright for the publications made accessible via the Queen's University Belfast Research Portal is retained by the author(s) and / or other copyright owners and it is a condition of accessing these publications that users recognise and abide by the legal requirements associated with these rights.

\section{Take down policy}

The Research Portal is Queen's institutional repository that provides access to Queen's research output. Every effort has been made to ensure that content in the Research Portal does not infringe any person's rights, or applicable UK laws. If you discover content in the

Research Portal that you believe breaches copyright or violates any law, please contact openaccess@qub.ac.uk. 


\section{Accepted Manuscript}

Untar geted metabolomic analysis of human ser um samples associated with differ ent levels of red meat consumption: a possible indicator of type 2 dia betes?

Daniel Carrizo, Olivier P. Chevallier, Jayne V. Woodside, Sarah F. Brennan, Marie M. Cantwell, Geraldine Cuskelly, Christopher T. Elliott

PII: S0308-8146(16)31673-9

DOI: http://dx.doi.org/10.1016/j.foodchem.2016.10.056

Reference: FOCH 20044

To appear in: Food Chemistry

Received Date: 6 May 2016

Revised Date: 26 September 2016

Accepted Date: 12 October 2016

Please cite this article as: Carrizo, D., Chevallier, O.P., Woodside, J.V., Brennan, S.F., Cantwell, M.M., Cuskelly, G., Elliott, C.T., Untargeted metabolomic analysis of human serum samples associated with differ ent levels of red meat consumption: a possibleindicator of type2 diabetes?, Food Chemistry (2016), doi: http://dx.doi.org/ 10.1016/j.foodchem.2016.10.056

This is a PDF file of an unedited manuscript that has been accepted for publication. As a service to our customers we are providing this early version of the manuscript. The manuscript will undergo copyediting, typesetting, and review of the resulting proof before it is published in its final form. Please note that during the production process errors may be discovered which could affect the content, and all legal disclaimers that apply to the journal pertain. 


\title{
Untargeted metabolomic analysis of human serum
}

\section{samples associated with different levels of red meat}

\section{consumption: a possible indicator of type 2 diabetes?}

\author{
Daniel Carrizo ${ }^{1,4^{*}}$, Olivier P. Chevallier ${ }^{1}$, Jayne V. Woodside ${ }^{2}$, Sarah F. \\ Brennan $^{3}$, Marie M. Cantwell ${ }^{3}$, Geraldine Cuskelly ${ }^{1}$, Christopher T. Elliott ${ }^{1}$ \\ ${ }^{1}$ Institute for Global Food Security, Queen's University, Belfast, 18-30 Malone Road, BT9 \\ 5BN, Northern Ireland, United Kingdom.
}

${ }^{2}$ UKCRC Centre of Excellence for Public Health (Northern Ireland), Institute of Clinical Science B, Royal Victoria Hospital, Grosvenor Road, Belfast, BT12 6BJ, UK.

${ }^{3}$ Centre for Public Health, School of Medicine, Dentistry and Biomedical Science, Queen's University Belfast, Belfast, BT12 6BA, United Kingdom

${ }^{4}$ Astrobiology Centre (CSIC-INTA), Dept. of Planetology and Habitability, Torrejón de Ardóz 28850, Madrid, Spain

\section{*Corresponding Author}

Tel: +34 915206433, Fax +34 915201074, E-mail: dcarrizo@cab.inta-csic.es, carrizodan@gmail.com 


\section{ABSTRACT}

Red meat consumption has been associated with negative health effects. A study to identify biomarkers of meat consumption was undertaken using serum samples collected from combining high resolution mass spectrometry (UPLC-QTof-MS) and chemometrics. Using orthogonal partial last-squares discriminant analysis (OPLS-DA), multivariate models were created for both modes of acquisition (ESI-/ESI+) and red meat intake classes (YES/NO). In the serum samples, a total 3280 and 3225 ions of interest were detected in positive and negative modes, respectively. Of these, 62 were found to be significantly different $(p<0.05)$ between the two groups. Glycerophospholipids as well as other family lipids, such as lysophospholipids or sphingomyelin, were found significantly $(\mathrm{p}<0.05)$ different between yes and no red meat intake groups. This study has shown metabolomics fingerprints have the capability to identify potential biomarkers of red meat consumption, as well as possible health risk factors (e.g., key metabolic families related to the risk of development type 2 diabetes).

Keywords: Red meat consumption, biomarkers analysis, UPLC-QTof-MS, metabolomics, lipids, health risk factors, Diabetes 


\section{Introduction}

Meat consumption is an important contributor to dietary protein in omnivorous populations, and therefore has a potential impact on a range of nutritional and health outcomes. In many western countries, and increasingly worldwide, a typical lifestyle is characterized by a diet high in fat, calories and red meat, and low in fruits, vegetables and fiber. An increasing number of epidemiological studies have associated red and processed meat consumption with the development of the major chronic diseases of the Western world; diabetes, coronary disease and colon cancer (IARC 2015, Cross et al., 2007; Giovannucci et al., 1994; Kelemen, Kushi, Jacobs, \& Cerhan, 2005; Kontogianni et al., 2008, Song et al., 2004). Constituents of red meat that have been proposed to be responsible for these associations, include the fat content, fatty acid composition and the possible formation of carcinogenic compounds, such as heterocyclic amines (HCAs), by cooking meat at high temperatures (Bingham et al., 2002). Although there are many studies documenting these associations, the results generated have not always been consistent and there are several methodological issues which limit the strength of their findings. In the same way as risks to human health from excessive red meat consumption may result, there are many health benefits of consuming red meat based on its nutritional composition. Red meat intake has most often been assessed using food frequency questionnaires, diet records or measurements of others parameters, although this is unspecific (Roberts et al., 1991, Leiba et al., 2005). These assessment methods have also been used extensively for studies on different kinds of meat (red vs. white), and on the influence of cooking methods. Several attempts have been made to find reliable markers of meat intake. Such markers would ideally reflect specific compounds in meat that distinguishes its intake from other foods and food groups. The development of robust food biomarkers will help in 
better classifying an individual's dietary intake and, in turn, will improve the assessment of the relationship between diet, health and chronic disease (Heinzmann et al., 2010). In recent years there has been an increased interest in applying metabolomics for the discovery of biomarkers of dietary intake. Recent proteomic studies have shown that short chain fatty acids (such as butyrate) generated from microbial digestion of dietary fiber may have important protective functions against gut cancers (Tan et al., 2006). Any alteration of the physiological status can disrupt homeostasis, resulting in perturbations of the levels of endogenous biochemicals that are involved in key metabolic processes in the cells and tissues of an organism (Nicholson \& Wilson, 2003). To maintain homeostasis, and to adjust for the changes in tissue biochemistry, the composition of the body will be altered accordingly and lead to different and dynamic "metabotypes" that may not be observable from gross examination of the phenotype. Thus, monitoring perturbations in biofluid composition specifically due to diet preferences may yield valuable information regarding underlying molecular mechanisms. The quantitative measurement of the dynamic multiparametric metabolic response of living systems to pathophysiological stimuli or genetic modification is termed metabonomics (Nicholson et al., 1999) and largely relies on chemometric analysis of high resolution spectral data (e.g., NMR or Q-Tof-MS).

The combination of high resolution liquid chromatography-quadrupole time-of-flight-mass spectrometry (UPLC-QTof-MS) and chemometrics has not been previously applied for biochemically profiling serum samples in relation to red meat intake levels. The aim of this investigation was to assess the relative analytical power and potential usefulness of UPLCQTof-MS for studying the polar metabolite fingerprint present due to different levels of red meat intake and to identify potential biomarkers (metabolites) of consumption. 


\section{Materials and methods}

\subsection{Serum samples}

Serum samples were obtained from the research project FoodCAP funded by the World Cancer Research Fund (Grant ID 2010/255). As part of this project, a randomized dietary intervention study was conducted. Initially, participants were screened for habitual meat intake, via completion of a 7 day food diary. Participants were recruited via poster and email advertisement and through word-of-mouth, they were given full study information and screened for eligibility. Those under 18 years were excluded as were smokers, due to potential exposure to polycyclic aromatic hydrocarbons. Eligible participants provided written informed consent. They received a 7-day food diary and detailed instructions on how to record their dietary intake. Participants were asked to record their dietary intake for seven consecutive days and to return within two days of completing their diary. Those in the top $33 \%$ of habitual meat intake were re-contacted and re-consented to take part in six week dietary intervention study. They were randomly allocated (using www.randomisation.com) to (i) remove all meat from their diet (high red meat intake (HRMI) group), or (ii) continue with their usual diet (control group), for 6 weeks. Each participant in the HRMI group was advised how to lower their meat for the intervention period. Participants were given personalized advice to achieve this and were instructed to employ food substitutions, with due recognition to the individuals' usual dietary habits and personal food likes and dislikes. Where appropriate, participants were provided with a choice of meat free meals (commercially available branded products) to assist them in complying with the intervention. Participants were asked to complete a second food diary mid-way through intervention as a record of actual food consumption during intervention. Where issues of compliance arose, they were 
addressed at this mid-way point. The control group continued to consume their usual diet. They provided blood samples and completed questionnaires as for HRMI group participants.

\subsection{Blood collection}

Participants were instructed to fast (water only allowed) for at least $12 \mathrm{~h}$ prior to attending each study visit at baseline (t0), after three weeks ( $\left.\mathrm{t}_{1}\right)$ and after six weeks (t2). Participants provided a $50 \mathrm{ml}$ blood sample, collected in anticoagulant plasma tubes and serum tubes, by staff trained in venepuncture. Serum tubes were allowed to coagulate at room temperature for approximately $1 \mathrm{~h}$, while plasma tubes were refrigerated. Serum and plasma were obtained by centrifugation, and were aliquoted and stored at $-80^{\circ} \mathrm{C}$. Each completed food diary was reviewed at the time of collection with a researcher to ensure correct completion. Analysis of food diaries (examining food intake and also through analysis of nutrient intake using the dietary analysis program WISP v3.0 (Tinuviel, UK), confirmed compliance with the dietary intervention. A total of 31 participants completed the intervention.

This study was conducted according to the guidelines laid down in the declaration of Helsinki and all procedures involving human subjects were approved by the School of Medicine, Dentistry and Biomedical Sciences Research Ethics Committee of Queen's University, Belfast.

\subsection{Sample extraction}

Extracted serum samples for metabolomics analysis (polar metabolites) were obtained using a minimal sample preparation method. Briefly, for each intervention sample a $100 \mu 1$ aliquot of serum was transferred into a $2 \mathrm{ml}$ sterile Eppendorf tube then $400 \mu \mathrm{l}$ of ice cold methanol were added. The samples were mixed $(5 \mathrm{sec})$ in a vortex, and then centrifuged for 
$15 \mathrm{~min}$ at $16,000 \mathrm{~g}$ at $4^{\circ} \mathrm{C}$. The supernatant layer was transferred to another $2 \mathrm{ml}$ Eppendorf tube and vacuum dried overnight. Dry extract were then reconstituted into $100 \mu \mathrm{l}$ of milli-Q water, transferred in to a $2 \mathrm{ml}$ Spin-X Centrifuge tube filter (Corning Incorporated, Corning, NY 14831, USA) and centrifuged for $15 \mathrm{~min}$ at $16,000 \mathrm{~g}$ at $4^{\circ} \mathrm{C}$. Filtered extract were then transferred to a $2 \mathrm{ml}$ Square Collection Plate (Water, Milford, MA, USA) for UPLC-QTofMS analysis.

\subsection{UPLC-QTof-MS analysis}

30 samples were analyzed (in triplicate, $n=90$ ), from $t_{0}, t_{1}$ and $t_{2}$ (high red meat intake $\mathrm{n}=48$, no red meat intake $\mathrm{n}=45$ ). A UPLC I-Class system (Waters Acquity, Milford, MA, USA) was used for chromatographic purposes, coupled to a mass spectrometer (Waters Xevo G2S QTof mass spectrometer (Manchester, UK) equipped with an ESI (electrospray ionization source) with lock-spray interface for accurate mass correction in real time. The source temperature and cone gas flow were set at $120^{\circ} \mathrm{C}$ and $50 \mathrm{l} / \mathrm{h}$, respectively. Meanwhile, desolvation temperature and gas flow were set up at $450^{\circ} \mathrm{C}$, and $850 \mathrm{l} / \mathrm{h}$, respectively. The capillary voltage was set at $1.0 \mathrm{kV}$ and $1.5 \mathrm{kV}$ in ESI+ and ESI-, respectively with a cone voltage of $30 \mathrm{~V}$ operating in both mode. Leucine Enkephalin $\left(2 \mathrm{ng} \cdot \mu \mathrm{l}^{-1}\right)$ in methanol/water with $0.1 \%$ formic acid $(1: 1, \mathrm{v} / \mathrm{v})$ was used as a lock mass solution, which was infused into the MS with a flow rate of $5 \mu 1 . \mathrm{min}^{-1}$. Mass spectra data were acquired in continuum mode using $\mathrm{MS}^{\mathrm{E}}$ function (in low energy: $4 \mathrm{eV}$ and high energy: ramp from 20-35 eV) over the range $\mathrm{m} / \mathrm{z}$ 50-1200 range with a scan time of 0.08 s. A $5.0 \mu \mathrm{l}$ of the sample was injected onto an Acquity UPLC HSS T3 column ( 2.1 x $100 \mathrm{~mm}, 1.8 \mu \mathrm{m}$, Waters, Milford,USA) mounted with a guard column (HSS T3 VanGuard Pre-column, $2.1 \mathrm{~mm}$ x $5 \mathrm{~mm}, 1.8 \mu \mathrm{m}$, Waters, Milford, MA, USA). The column oven and the sample manager temperatures were $45^{\circ} \mathrm{Cand} 10^{\circ} \mathrm{C}$, respectively. The elution buffers were A (water with $0.1 \%$ formic acid) and B (methanol with 
$0.1 \%$ formic acid), with a flow rate of $0.4 \mathrm{ml} \cdot \mathrm{min}^{-1}$. The elution gradient (A:B, v/v) was: 2 min at 99:1 isocratic, followed by a linear gradient from 99:1 to 15:85 over 4 min then a linear change from 15:85 to 10:90 over 3 min and finally a linear gradient from 10:90 to 1:99 over $7 \mathrm{~min}$. After a $2 \mathrm{~min}$ period at 1:99, initial composition 99:1 was restored and held for 2 min before the next injection. Samples were injected in triplicate in negative (ESI-) and positive (ESI+) ionization. For chromatographic performance (i.e., reproducibility of retention times and peak intensities) 10 pooled samples were injected prior to the analysis and every 10 target samples another small set of pooled samples (5) were injected from beginning to end of the run(Oresic et al., 2011).

\subsection{Data analysis}

The generated spectra data was handled using Progenesis QI v2.0 Software (Waters, Newcastle, UK), this is a specific software package for metabolomics analysis that permit to accurately treat high resolution LC-MS spectral data. This software assisted in the validation of LC-MS approaches and identification of features within the spectral data. Using Progenesis QI the spectral data were aligned to a chosen pooled sample, adduct ions were deconvoluted and ion abundance above the threshold level performed. All ions detected were selected against the Lipid Blast database, Chemspider and Progenesis Metascope spectral libraries which provide identifications for $15-30 \%$ of the assigned ions. The analyzed data was exported to Simca 14 (Umetrics, Umea, Sweden) for multivariate analysis. Data quality was evaluated in terms of reproducibility using an approach utilize in others works by Spagou et al., 2011. As a quality control estimate all the spectral data were mean centered, pareto scaled and analyzed with principal components analysis (PCA). For both ESI+ and ESIacquisition modes, the data were divided into high red meat intake and no red meat intake prior to analysis using orthogonal projection to latent structures-discriminant analysis (OPLS- 
DA). Red meat intake was analyzed for two different levels of consumption (yes and no) at different times during the intervention study, the initial baseline samples $\left(\mathrm{t}_{\mathrm{o}}\right)$ and the post intervention samples at 6 weeks $\left(t_{2}\right)$. Statistical analysis approach was performed following the proposed minimum reporting standards for data analysis in metabolomics by Goodacre et al., 2007. More details about statistical models used can be found in the Supporting Information material and in an earlier work by Graham et al., 2013. In each of the models $(n=2)$, all of the test set observations were correctly assigned to exposure levels (yes and no) groups $100 \%$ of the time for both ESI+ and ESI- (available in Figures S1 and S2, Supporting Information). Target ions which were different between groups (high and no red meat intake) were analyzed using a two tailed student t-test. The top 100 compounds responsible for the fluctuation in the discriminant model were picked from the variable importance plot (VIP) in the two cases, and subjected to univariate analysis (the same procedure were applied for the initial intervention samples,$t_{0}$ in order to compare similar ions (i.e., compounds of interest) in both groups).

\section{Results and discussion}

\subsection{Characteristics of the population studied}

The average age of participants was 30.5 years in both men $(n=16)$ and women $(n=14)$. About $6 \%$ were obese $\left(B M I \geq 30 \mathrm{~kg} / \mathrm{m}^{2}\right)$. The group for red meat intake comprised of 14 individuals and the control group had 16 individuals. No significant differences $(p>0.05)$ were found between groups for BMI $\left(18.16 \mathrm{~kg} / \mathrm{m}^{2}\right.$ and $18.07 \mathrm{~kg} / \mathrm{m}^{2}$ and age 30.2 years and 31.1 years, respectively).

\subsection{Red meat intake models of exposure}




\subsubsection{Models of Exposure at the end of the study $\left(t_{2}\right)$}

Figure 1A shows a chromatogram acquired in ESI- for a target serum sample and the corresponding pool serum sample (pink and green line, respectively). Orthogonal partial least-squares discriminant analysis (OPLS-DA) was used to develop a model enabled to differentiate between no red meat intake (blue) and high red meat intake (red), in serum samples $(n=75)$. Figure 1B shows the score plot with the results of the model building, with one component and eight orthogonal projections which demonstrate the highest load of variation between the two groups $(\mathrm{R} 2(\mathrm{cum})=97 \%$; $2=86 \%$; root mean squared error for validation $(\mathrm{RMSEV})=18 \%$. Figure $1 \mathrm{C}$ displays the loading plots correlated to the scores plot in Figure 1B (reveals the compounds that are the reason for the variation between the two samples groups). Featured in blue (no red meat intake, $\mathrm{n}=15$ ) and red (high red meat intake, $\mathrm{n}=15$ ) were the ions which shows significant differences between sample groups. The S-plot in Figure 1D reveals the proportional importance of each molecule in discriminate sample groups. Peak height and colour point out relative significance of the candidate molecules. Table 1 shows the retention times, exact masses, percentage increment and their significance values $(\mathrm{p}<0.05)$. Figures $2 \mathrm{~A}$ and $2 \mathrm{~B}$ show the proportional abundance of all the ions (ESI-) of concern within the two sample groups (yes vs no red meat intake, respectively), including the standard error of the mean (error bars).

In Figure 3A a chromatogram acquired in positive mode of the sample and the corresponding pool serum sample (pink and green line, respectively) is shown. The same scheme was applied for data analysis as in the former described negative mode acquisition, and 3225 ions were detected by Progenesis QI. The OLPS-DA scores model shown in Figure 3B $(n=83)$, was built with one component and eight orthogonal projections to elucidate the maximum amount of variation within the two sample groups (R2 (cum) $=94 \%$; Q2 (cum) $=89 \%$; RMSEV $=17 \%$ ). Figure $3 \mathrm{C}$ shows the loading plots in accordance to the compounds that are 
accountable for the variation between the two samples groups. Displayed in blue (high red meat intake, $n=15$ ) were the ions which showed significant differences between groups, no significant differences in compounds were observed for no red meat intake. Figure 3D shows the significance and relative importance of the ions found in Fig.3C. Table 2 remarks the retention times, masses, percentage of increase between sample groups, and their significance values (only ions with $\mathrm{p}<0.05$ are shown) of the ions. Figure $4 \mathrm{~A}$ shows the relative abundance of all the ions (ESI+) of interest between the two sample groups (high red meat intake), including the standard error of the mean (error bars). Models of exposure at the beginning of the study $\left(\mathrm{t}_{0}\right)$ can be found in the SI material.

\subsection{Biomarker identification}

Progenesis QI provided 3075 possible identifications for the 3400 ions found to be present in the target samples extracts ( $t_{2}$ post intervention samples). Nevertheless only a small number of these features were presumed identified using Progenesis QI, with only 32 (ESI+) and 30 (ESI-) being in the top 100 metabolites emphasize by the VIP (Variable Importance Plot). The tentatively identified compounds which may be candidates for probable metabolite biomarkers of red meat intake, need to be interpreted with a degree of caution as the identification is not $100 \%$ accurate. We followed the recommendations for standard metabolite identification established by the Chemical Analysis Working Group (CAWG) of the Metabolomics Standard Initiative by Sumner et al. 2007. Therefore we describe our identified compounds as putatively annotated compounds (type 2) or putatively characterized compound class (type 3 ). In addition, for a specific $\mathrm{m} / \mathrm{z}$ more than one possible compound can be listed as a possible candidate. Further research is required to compare the fractionation patterns and retention times of the potential identified compounds against reference standards using UPLC-MS/MS. Within the tentatively identified compounds, we were able to reveal 
important lipids families (e.g. Glycerophospholipids), and within this class: Glycerophosphocholines (GP01) and Glycerophosphoethanolamines (GP02), and subclasses: $\quad$ Monoacylglycero-phosphocholines

(GP0105) and $1 Z-$ alkenylglycerophosphoethanolamines (GP0207), as well other important lipid families, such as Lysophospholipids, Phosphatidylcholine or Sphingomyelin, were identified and found significantly different between groups, some were upregulated and others down regulated $(\mathrm{p}<0.05)$ (Figures 2A, 2B, 4, S5, S6 and S7). Glycerophosphocholines have previously been associated with a "meat factor" which is reported to enhance non-heme iron absorption (Armah et al., 2008), this compound could be used as a positive biomarker of red meat intake. For the VIP plot, 32 compounds were significantly different $(\mathrm{p}<0.05)$ in the ESI+, for the high red meat intake group, surprisingly no compounds were significantly different in the 'no red meat' intake group. For the VIP plot, 15 compounds were significantly different $(\mathrm{p}<0.05)$ in the ESI-, between the high red meat intake group vs the no red meat intake group and 15 compounds between the no red meat intake and the high red meat intake group. For the 62 compounds, which were significantly different from high vs no exposure, 18 were positively "identified". Table S1 shows the presumptive identifications of these 18 compounds.

Three compounds $(\mathrm{m} / \mathrm{z}$ 464.3140- $\mathrm{PE}(\mathrm{P}-18: 0 / 0: 0) ; \mathrm{m} / \mathrm{z}$ 724.5279- $\mathrm{PE}(\mathrm{P}-16: 0 / 20: 3)$ and $\mathrm{m} / \mathrm{z}$ 726.5447- PE(18:1(9Z)/18:1(9Z)) which are related to the Glycerophosphoethanolamines (GP02) specific to the 1Z-alkenylglycerophosphoethanolamines (GP0207) (ID level type 2 or type 3, Table S1), were found for ESI- mode in high red meat intake, these compounds have two different origins (endogenous and food) and have been linked to have a cell signaling, energy source/storage function, as well as being components of cell membranes (www.hmbd.ca). Other compounds identified as being significantly higher in the red meat intake group were Sphingomyelin d18:C18:0, $(\mathrm{m} / \mathrm{z}$ 775.5959) and Phosphatidylserine 18:018:1, ( $\mathrm{m} / \mathrm{z}$ 786.5647) (ID level type 3, Table S1). Sphingomyelin altered levels have been 
shown to be markers of a number of different metabolic syndromes (e.g., obesity, insulin resistance of fatty liver disease) (Hanamatsu et al., 2014). Phosphatidylserines have different biological functions in the human body, such as cell signaling membrane component and energy storage (www.hmbd.ca).

Seven compounds $(m / z$ 517.3173- PC(18:3/0:0); $m / z$ 519.3321- PC(18:2/0:0); $m / z$ 545.3487PC(20:3/0:0); $m / z$ 729.5316-GPCho(6:0/26:2); $m / z$ 743.5481- GPCho(16:0/17:2); $m / z$ 782.5708- GPCho(14:0/22:4) and $m / z$ 799.6692), all belonging to the Glycerophospholipids class (GP01) and Monoacylglycero-phosphocholines sub-class (GP0105) were found in ESI+ mode in the high red meat intake group (ID level type 2 or type 3, Table S1). This family of compounds has been previously reported as potential biomarkers of red meat consumption and was related to a higher risk of type 2 diabetes (Wittenbecher et al., 2015). Within the presumptive 18 identified compounds in Table S1 (ID level type 2 or type 3), 16 were upregulated in the HRMI group compared to the control.

This is the first study that combines high resolution UPLS-QTof-MS and chemometrics to biochemically profile the polar metabolome in human serum samples in an effort to evaluate diet preferences (red meat intake yes or not). By acquiring MS data in negative and positive modes, two separate metabolomics models were developed which can clearly and unambiguously distinguished high vs low levels of contaminant exposure. The predictive accuracy of the models were excellent with a range of Q2= 77 - 93\% and RMSEV, $12-18 \%$. The predictability of the model could be increased if a larger number of samples are measured. Data acquired in positive mode assigned a total of 3076 polar compounds while data acquired in negative mode assigned 3121 polar compounds. It has been possible to assess the variable importance of a number of ions which were highlighted by their respective colours in the S-line-plot. The S-plot allowed the visual determination of the ions of interest most responsible for separating the two study groups (high vs no red meat intake). It was then 
possible to statistically analyze each of the ions of interest and verify (by means of a $t$-test) that these candidates were significantly different in each level of exposure (Table 1 and 2). In order to search for possible metabolite correlations between the initial $\left(\mathrm{t}_{0}\right)$ and the final intervention samples after the six weeks study $\left(\mathrm{t}_{2}\right)$, the VIP plot ions (100) for ESI-/ESI+ and red meat intake groups were investigated. For the 400 ions analyzed only 38 ions were significantly different within both study groups (figure S5, S6 and S7, SI material). An additional comparison of these ions within $t_{2}$ and $t_{0}$ samples, shows that only 8 of these ions were also significantly different between the two groups analyzed for the last samples of the intervention study $\left(\mathrm{t}_{2}\right)$. The tentative identification of these compounds can be found in Table S2. A number of other observational studies have used metabolomic (NMR spectroscopy) analysis of serum or urine samples to examine associations with red meat intake (Stella et al., 2006; Bertram et al., 2007a, 2007b), showing some similarities in results for biomarkers identified (e.g., Glycerophospholipids), these support the findings of the present study. Within the assigned compounds in both modes, 62 compounds showed significant differences $(\mathrm{p}<0.05)$, in the ESI+ mode, 32 compounds were found for the high red meat intake group, but surprisingly none were significantly different (between groups) in the no red meat intake group (Table 1 and 2, only show the top 15 of each group (high red meat and no red meat intake) and for both acquisition modes, 45 ions in total). For the ESI- mode 15 compounds were significantly different $(\mathrm{p}<0.05)$ for the high red meat intake group and 15 compounds for the no red meat intake, encompassed important lipid families (e.g. Glycerophosphoethanolamines (GP02) or Glycerophospholipids (GP01)). This demonstrates the strength of this method for tracking biochemical changes in serum samples in response to dietary change. We were able to positively identify (as putative annotated compounds type 2 or putative characterized compound classes type 3) important lipids classes, such as Glycerophosphocholines (GP01) and Glycerophosphoethanolamines (GP02), 
Lysophospholipids, Phosphatidylcholine, Sphingomyelin or Monoacylglycerophosphocholines, which are involved in lipid metabolism (cell signaling, fuel storage/source or membrane function/component).

\section{Conclusions}

In the present study an effect of changing red meat intake on the levels of a range of different lipids families was found and these, or at least a number of them, have the potential to be used as positive biomarkers of red meat consumption (i.e., Glycerophosphocholines (GP01). The data generated shows that red meat consumption contributed to a rather different metabolic fingerprint among the study population. A deregulated lipidomic profile, through the enrichment (up-regulated) of lipid metabolites that contain 16 or more carbons atoms in the fatty acid residues in which one fatty acid was contained (lysophospoatidylcholines and sphingomyelin) or 34 or more carbon atoms in which 2 fatty acids were contained (diacyl phosphatidylcholines and acyl-alkyl phosphatidylcholines) is plausibly related to the high proportion of even-numbered long-chained fatty acids contained in red meats (Williams, 2007). Several studies have related altered lipid metabolism to the pathogenesis of type 2 diabetes, among them a challenged mitochondrial metabolism, altered cellular signaling processes at the membrane, and modulation of gene expression (Ye, 2013; Amati, 2012). Thus, using the data in Table S2, we proposed a ratio of compounds as a possible biomarker index of red meat intake. We used the sum of three compounds (a lysophosphatidylcholine, LysoPC(18:2(9Z,12Z)), a sphingomyelin SM(d18:1/16:0) and a diacyl phosphatidylcholine PC(20:4(5Z,8Z,11Z,14Z)/16:0) at the beginning of the study $\left(\mathrm{t}_{0}\right)$ and at the end of the study $\left(t_{2}\right)$. The ratios for the "biomarker index" was 0.97 for the $t_{0}$ samples and 1.23 for the $t_{2}$ samples, this result is in agreement (an increasing ratio towards the end of the study) with what was expected, i.e. a higher metabolomic difference between both groups over the 
experiment time. Thus, serum levels of multiple lipids can subtly reflect the status of a metabolic system in response to a change in food group intake, in this case red meat consumption (e.g., through the id compounds and the "biomarker ratio") as well as possible health risk factors (e.g., key metabolic families related to the risk of development diabetes 2 type).

\section{Acknowledgments}

This work was kindly supported by the World Cancer Research Fund. D. Carrizo acknowledges Ministerio de Economía y Competitividad (Spain) for the financial support through a Ramón y Cajal (RYC) Contract (RYC-2014-19446).

\section{References}

Armah, C.N.; Sharp, P.; Mellon, F.A.; PAraigh, S.; Lund, E.K.; Dainty, J.R.; Teucher, B.; Fairweather-Trait, S.J. L- $\alpha$-Glycerophosphocholine Contributes to Meat's Enhancement of Nonheme Iron Absorption. J. Nutr. 2008, 138, 873-877.

Amati, F. Revisiting the diacylglycerol-induced insulin resistance hypothesis. Obes Rev, 2012, 13(Suppl 2):40-50.

Bertram, H. C.; Hoppe, C.; Petersen, B.O.; Duus, J.O.; Molgaard, C.; Michaelsen, K.F. An NMR-based metabolomic investigation on effects of milk and meat protein diets given to 8year-old boys. British Journal of Nutrition, 2007a, 97:758-763.

Bertram, H. C.; Malmendal, A.; Petersen, B.O.; Madsen, J.C.; Pedersen, H.; Nielsen, N.C., et al. Effect of magnetic field strength on NMR-based metabolomic human urine data. 
Comparative study of 250, 400, 500, and 800 MHz. Analytical Chemistry, 2007b, 79: 71107115.

Bingham, S.A., Hughes, R, Cross, A.J. Effect of white versus red meat on endogenous Nnitrosation in the human colon and further evidence of a dose response. $J$ Nutr, 2002, 132, $3522 \mathrm{~S}-3525 \mathrm{~S}$.

Cross, A. J., Leitzmann, M. F., Gail, M. H., Hollenbeck, A. R., Schatzkin, A., \& Sinha, R. A prospective study of red and processed meat intake in relation to cancer risk. PLos Medicine, 2007, 4, 1973.

Eriksson, L. 2006. Multi- and Megavariate Data Analysis, Umetrics AB.

Giovannucci, E., Rimm, E. B., Stampfer, M. J., Colditz, G. A., Ascherio, A., \& Willet, W. C. Intake of fat, meat and fiber in relation to risk of colon cancer in men. Cancer Research, 1994, 54, 2390-2397.

Goodacre, R., Broadhurst, D., Smilde, A.K. et al. Proposed minimum reporting standards for data analysis in metabolomics. Metabolomics, 2007, 3, 231-241.

Graham, S.F., Chevallier, O.P., Roberts, D., Holscher, C., Elliot, C., Green, B.D. Investigation of the human brain metabolome to identified potential markers for early diagnosis and therapeutic targets of Alzheimer's disease. Anal. Chem, 2003. 85, 1803-1811.

Hanamatsu, H.; Ohnishi, S.; Sakai, S.; Mitsutake, S.; Takeda, H.; Hashino, S.; Igarashi, Y. Altered levels of serum sphingomyelin and ceramide containing distinct acyl chains in young obese adults. Nutrition \& Diabetes, 2014, 4: e141. 
Heinzmann, S.S., Brown, I.J., Chan, Q., Bictash, M., Dummas, M-E, et al. Metabolic profiling strategy for discovery of nutritional biomarkers: proline and betaine as a marker of citrus consumption. Am. J Clin. Nutr. 2010, 92, 436-443.

International Agency for Research on Cancer. Volume 114: Consumption of red meat and processed meat. IARC Working Group. Lyon; 6-13 September, 2015. IARC Monogr Eval Carcinog Risks Hum (in press).

Kelemen, L. E., Kushi, L. H., Jacobs, D. R., Jr, \& Cerhan, J. R. Associations of dietary protein with disease and mortality in a prospective study of postmenopausal women. American Journal of Epidemiology, 2005, 161, 239-249.

Kontogianni, M. D., Panagiotakos, D. B., Pitsavos, C., Chrysohoou, C., \& Stefanadis, C. Relationship between meat intake and the development of acute coronary syndromes: The CARDIO2000 case-control study. European Journal of Clinical Nutrition, 2008, 62, 171177

Leiba, A.; Vald, A.; Peleg, E.; Shamiss, A.; Gross, A. E. Does dietary recall adequately assess sodium, potassium, and calcium intake in hypertensive patients? Nutrition, 2005, 21, 462-466.

Nicholson, J.K., Wilson, I.D. Opinion: understanding 'global' systems biology: metabonomics and the continuum of metabolism. Nat rev Drug Discov, 2003, 2: 668-676.

Nicholson, J.K., Lindon, J.C., Holmes, E. 'Metabonomics': understanding the metabolic responses of living systems to pathophysiological stimuli via multivariate statistical analysis of biological NMR spectroscopic data. Xenobiotica, 1999, 29, 1181-1189.

Oresic, M.; Hyotylainen, T.; Herukka, S.; Sysi-Aho, M.; Mattila, I.; Seppanan-Laakso, T.; Julkunen, V.; Gopalacharyulu, P.; Hallikainen, M.; Koikkalainen, J.; Kivipelto, M.; Helisalmi 
S.; Lotjonen, J.; Soininen, H. Metabolome in progression to Alzheimer's disease. Transl. Psychiatry. 2011, DOI: 10.1038/tp.2011.55.

Roberts, S. B.; Ferland, G.; Young, V. R.; Morrow, F. M.; Heyman, M. B.; Melanson, K.; G. E., D. Objective verification of dietary intake by measurement of urine osmolality. Am. J. Clin. Nutr, 1991, 54, 774-782.

Song, Y., Buring, J.E., Manso, J.E., Liu, S. A prospective Study of Red Meat Consumption and Type 2 Diabetes in Middle-Ages and Elderly Women. Diabetes Care, 2004, 27, 21082115.

Tan, H.T., Zubaidah, R.M., Tan, S., Hooii, S.C., Chung, M.C.M. 2-D DIGE analysis of butyrate-treated HCT-116 cells after enrichment with heparin affinity chromatography, $J$ Proteome Res, 2006, 5: 1098-1106.

Spagou, K.; Wilson, I.; Masson, P.; Theodoridis, G.; Raikos, N.; Coen, M.; Holmes, E.; Lindon, J.C.; Plumb, R.S.; Nicholson, J.K.; Want, E.J. HILIC-UPLC-MS for exploratory urinary metabolic profiling in toxicological studies. Anal. Chem. 2011, 83, 382-390.

Stella, C.; Beckwith-Hall, B.; Cloarec, O.; Holmes, E.; Lindon, J. C.; Powell, J., et al. Susceptibility of human metabolic phenotypes to dietary modulation. Journal of Proteome Research, 2006, 5: 2780-2788.

Tsang, T.M.; Haselden, J.N.; Holmes, E. Metabonomic characterization of the 3nitropropionic acid rat Model of Huntington's disease. Neurochem, Res. 2009, 34, 12611271.

The Human Metabolome Database (HMDB). www.hmbd.ca

Williams, P. Nutritional composition of red meat. Nutr Diet, 2007, 64 (Suppl 4):S113-9. 
Wittenbecher, C.; Muhlenbruch, K.; Kroger, J.; Jacobs, S.; Kuxhaus, O.; Floegel, A.; Fritsche, A.; Pischon, T.; Prehn, C.; Adamski, J.; Joost, H.G.; Boeing, H.; Schulze, M.B. Amino acids, lipid metabolites, and ferritin as potential mediators linking red meat consumption to type 2 diabetes. Am J Clin Nutr, 2015, doi: 10.3945/ajcn.114.099150.

Ye, J. Mechanisms of insulin resistance in obesity. Front Med, 2013, 7:14-24. 
Table 1. Molecules of interest as identified in the S-Line Plot (ESI-), for the significance to the $\mathrm{Model}^{+}$

\begin{tabular}{|c|c|c|c|c|c|c|c|c|c|}
\hline \multicolumn{10}{|c|}{ negative mode acquisition } \\
\hline \multicolumn{5}{|c|}{ higher in high red meat intake } & \multicolumn{4}{|c|}{ higher in no red meat intake } & \\
\hline $\mathbf{n}^{\mathbf{0}}$ & Rt & mass & $\%$ increase & p-value & $\mathbf{n}^{0}$ & $\mathbf{R t}$ & mass & $\%$ increase & p-valu \\
\hline 1 & 5.33 & 255.0877 & 270.1 & $5.8 \times 10^{-04}$ & 1 & 4.16 & 171.0116 & 625.34 & $2.6 \times 10^{-09}$ \\
\hline 2 & 5.17 & 1012.4874 & 349.9 & $8.5 \times 10^{-04}$ & 2 & 5.60 & 385.1690 & 208.3 & 0.005 \\
\hline 3 & 6.41 & 367.1585 & 154.5 & $7.4 \times 10^{-03}$ & 3 & 5.71 & 462.1766 & 307.5 & $1.9 \times 10^{-05}$ \\
\hline 4 & 6.86 & 369.1734 & 160.1 & 0.01 & 4 & 6.75 & 624.3390 & 180.4 & 0.009 \\
\hline 5 & 6.87 & 397.2054 & 141.1 & 0.01 & 5 & 6.97 & 481.3521 & 188.3 & 0.00021 \\
\hline 6 & 7.10 & 448.3060 & 51.5 & 0.004 & 6 & 7.02 & 567.3163 & 429.3 & $1.2 \times 10^{-06}$ \\
\hline 7 & 8.61 & 479.3344 & 121.9 & 0.001 & 7 & 7.32 & 471.2425 & 24.2 & 0.02 \\
\hline 8 & 8.63 & 437.2899 & 133.3 & 0.004 & 8 & 7.33 & 621.3271 & 1605.2 & 0.0005 \\
\hline 9 & 9.22 & 327.2325 & 137.7 & 0.03 & 9 & 7.50 & 392.2923 & 302.5 & 0.007 \\
\hline 10 & 9.71 & 464.3140 & 137.7 & 0.001 & 10 & 7.57 & 391.2847 & 351.7 & 0.007 \\
\hline 11 & 14.95 & 485.2724 & 121.6 & 0.01 & 11 & 7.60 & 481.3521 & 154.6 & 0.0008 \\
\hline 12 & 15.69 & 775.5959 & 122.1 & 0.003 & 12 & 9.35 & 595.2888 & 70.8 & 0.006 \\
\hline 13 & 15.91 & 786.5647 & 124.3 & 0.004 & 13 & 14.20 & 824.5444 & 67.8 & 0.01 \\
\hline 14 & 16.08 & 724.5279 & 132.2 & 0.03 & 14 & 15.36 & 738.5071 & 78.1 & 0.02 \\
\hline 15 & 17.01 & 726.5447 & 146.3 & 0.0008 & 15 & 15.62 & 764.5234 & 63.4 & 0.005 \\
\hline
\end{tabular}

${ }^{+}$Accompanying are the respective results from the Student's t-test carried out for the high red meat intake level compared to low red meat intake. The results correspond to the S-line plot in Figure 1 which are numbered accordingly.

Table 2. Molecules of interest as identified in the S-Line Plot (ESI+), for the significance to

\begin{tabular}{|c|c|c|c|c|c|c|c|c|c|}
\hline \multicolumn{10}{|c|}{ positive mode acquisition } \\
\hline \multicolumn{10}{|c|}{ higher in high red meat intake } \\
\hline $\mathbf{n}^{0}$ & Rt & mass & $\%$ increase & p-value & $\mathbf{n}^{0}$ & Rt & mass & $\%$ increase & p-value \\
\hline 1 & 7.55 & 517.3173 & 165.9 & 0.0005 & 9 & 13.30 & 674.5371 & 134.2 & 0.0003 \\
\hline 2 & 7.73 & 240.6845 & 149.9 & 0.0008 & 10 & 13.66 & 700.5527 & 129.9 & 0.0003 \\
\hline 3 & 7.74 & 1039.6697 & 212.7 & 0.0002 & 11 & 13.94 & 689.5601 & 128.1 & 0.001 \\
\hline 4 & 7.91 & 519.3321 & 144.8 & 0.001 & 12 & 14.23 & 729.5316 & 141.9 & 0.002 \\
\hline 5 & 7.97 & 1015.6722 & 144.2 & 0.009 & 13 & 14.83 & 743.54810 & 130.8 & 0.0006 \\
\hline 6 & 8.25 & 545.3487 & 141.3 & 0.004 & 14 & 14.97 & 782.5708 & 139.2 & 0.001 \\
\hline 7 & 12.00 & 646.5051 & 148.9 & 0.001 & 15 & 17.79 & 799.6692 & 123.7 & 0.001 \\
\hline 8 & 12.36 & 673.5283 & 151.8 & 0.00003 & & & & & \\
\hline
\end{tabular}

the $\mathrm{Model}^{+}$

\footnotetext{
${ }^{+}$Accompanying are the respective results from the Student's t-test carried out for the high red meat intake levels compared to low red meat intake. The results correspond to the S-line plot in Figure 3 which are numbered accordingly.
} 


\section{Figure captions}

Figure 1. (A) UPLC ESI- chromatogram of the polar extract of a serum sample. (B) The scores plot displaying the separation between the two sample groups (no red meat intake $=$ blue; high red meat intake $=$ red). Explained variance $(\mathrm{R} 2)$ was $97 \%$, predictive ability $(\mathrm{Q} 2)$ was $86 \%$, and root-mean-square error of validation (RMSEV) was $18 \%$. (C) The loadings plots which correspond to the scores in B. Indicated in blue $(n=15)$ and red $(n=15)$ are metabolites which significantly differ between groups (higher in no red meat intake and high in high red meat intake, respectively). These are further emphasized by the S-line-plot (D) and their relative variable importance to the model.

Figure 2. Graphical representation of the mean relative abundances of the molecules found to be significantly different between sample groups acquired in negative mode for high red meat intake and no red meat intake. (A) shows which ions are at higher levels in high red meat intake samples compared to no red meat intake; (B) exhibits those ions higher in no red meat intake samples than in high red meat intake. Numbered columns relate to the ions labeled in Table 1.

Figure 3. (A) UPLC ESI+ chromatogram of the polar extract of a serum sample. (B) The scores plot displaying the separation between the two sample groups (no red meat intake $=$ red; high red meat intake $=$ blue $)$. Explained variance $(\mathrm{R} 2)$ was $94 \%$, predictive ability $(\mathrm{Q} 2)$ was $89 \%$, and root-mean-square error of validation (RMSEV) was $17 \%$. (C) The loadings plots which correspond to the scores in B. Indicated in blue $(\mathrm{n}=15)$ are metabolites which 
are metabolites which significantly differ between groups (higher in high red meat intake, no significant ions were found for no red meat intake group). These are further emphasized by the S-line-plot (D) and their relative variable importance to the model

Figure 4. Graphical representation of the mean relative abundances of the molecules found to be significantly different between sample groups acquired in positive mode for high red meat intake. (exhibit those ions higher in high red meat intake group samples than in no red meat intake), no significant different ions were found in the no red meat intake group). Numbered columns relate to the ions labeled in Table 2 for both levels of red meat intake. 


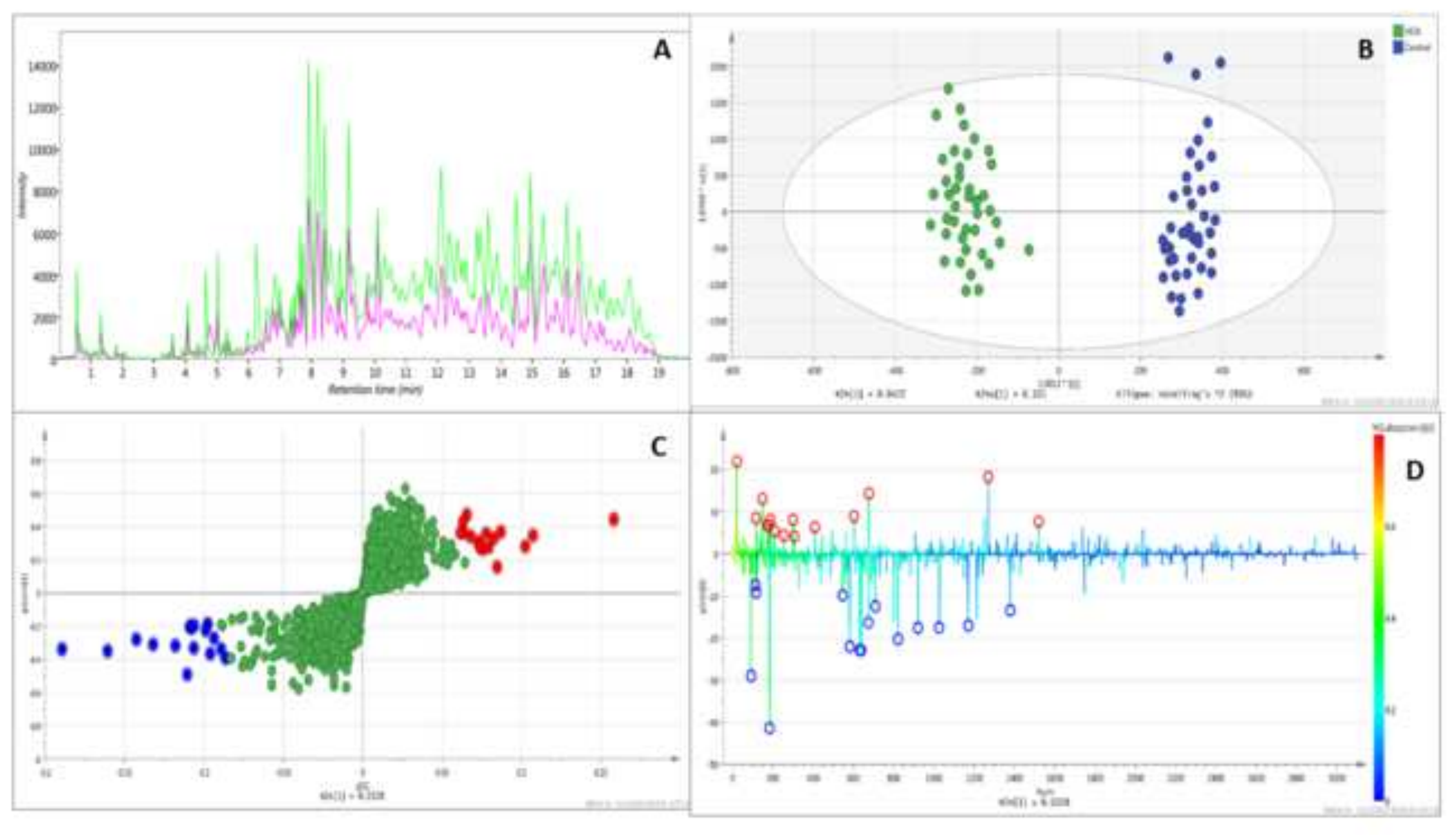

\section{ACCEPTED MANUSCRIPT}
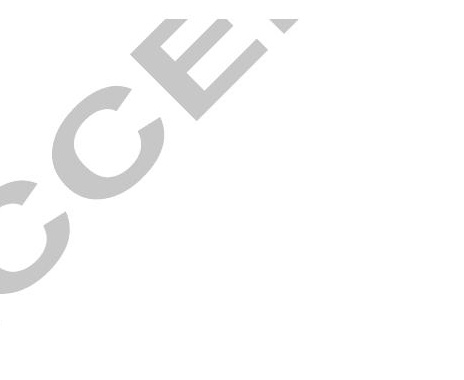


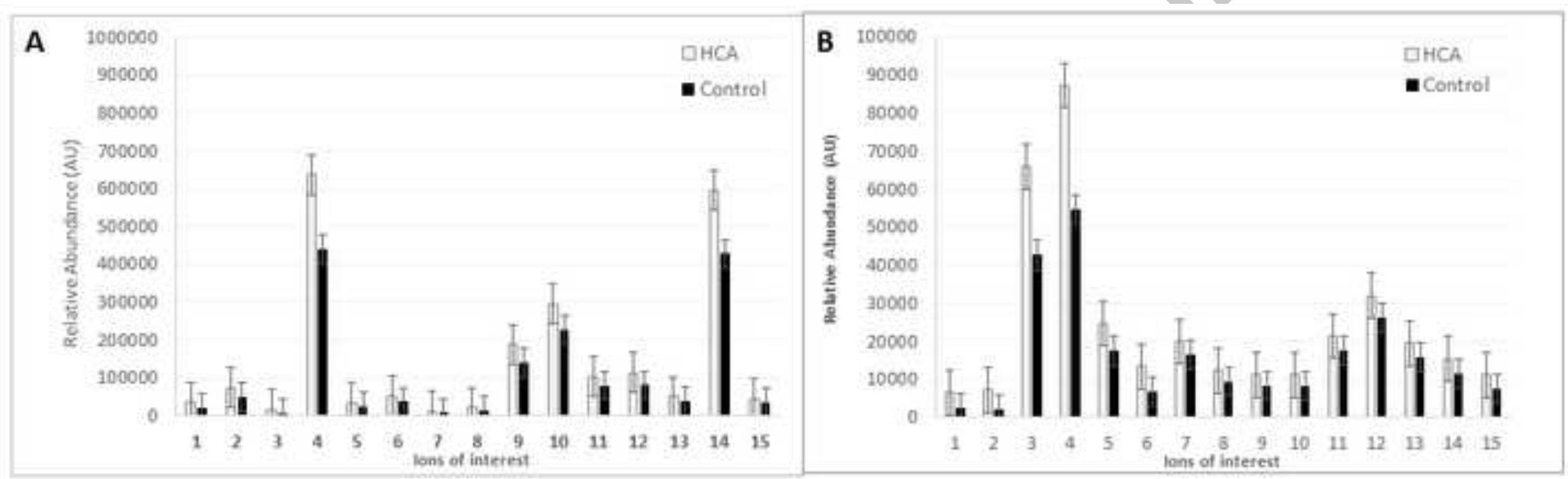



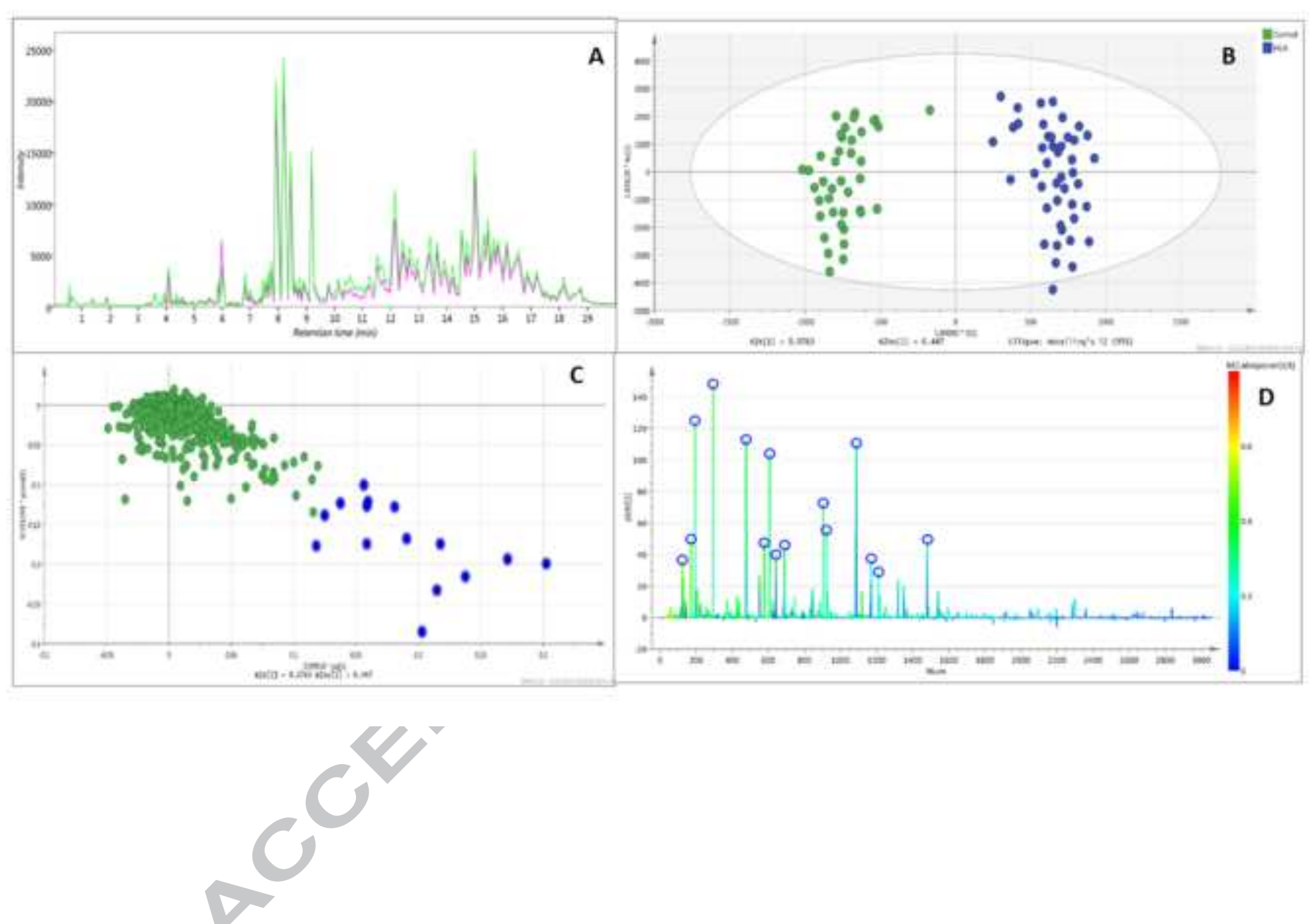 \\ ACCEPTED MANUSCRIPT}

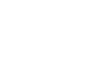




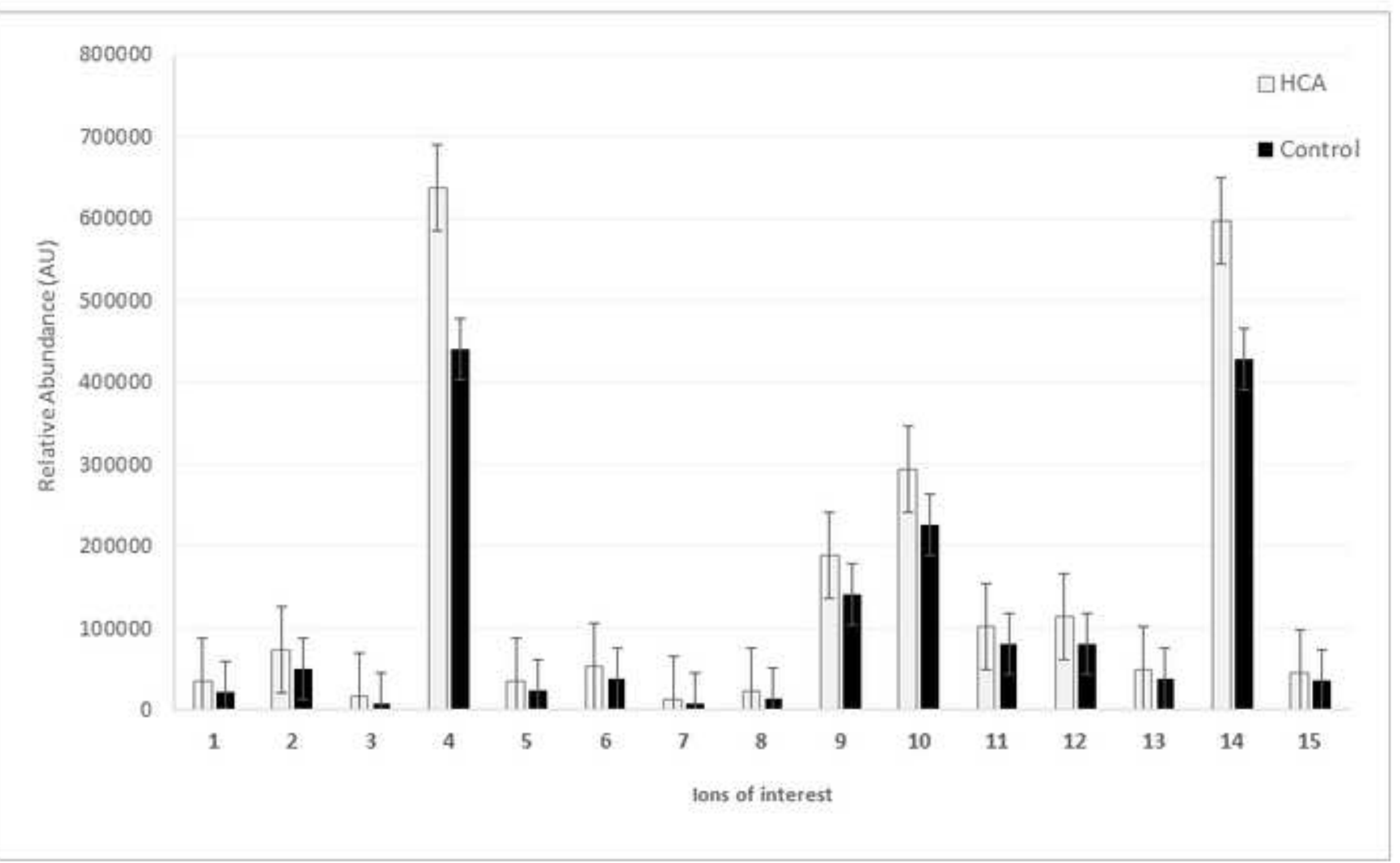


Highlights

- An intervention study was undertaken in health participants with a high red meat intake and no red meat intake for six weeks.

- Utilization of high resolution mass spectrometry (liquid chromatography-quadrupole time-of-light-mass spectrometry, UPLC-QTof-MS) and chemometrics for biomarkers identification.

- Identification of possible biomarkers of red meat consumption were found between groups.

- Positive identification of Type 2 Diabetes biomarkers were found in the high red meat intake group. 